\title{
Flea and tick treatment satisfaction, preference, and adherence of dog owners in the United States, United Kingdom, and Australia who treated their dog with fluralaner
}

\author{
Robert Lavan $^{1 *}$, Rob Armstrong ${ }^{2}$, Karen Lipworth ${ }^{3}$, Dorothy Normile² and Hannah Newbury ${ }^{4}$ \\ ${ }^{1}$ Outcomes Research, Merck Animal Health, Center for Observational and Real-World Evidence, Merck \& Co., Inc, \\ Kenilworth, NJ, USA \\ ${ }^{2}$ Merck Animal Health, Madison, NJ, USA \\ ${ }^{3}$ MSD Animal Health, Sydney, Australia \\ ${ }^{4}$ MSD Animal Health, Walton Manor, Walton, Milton Keynes, UK
}

\begin{abstract}
Background: An extended duration flea and tick medication of the isoxazoline class (fluralaner) was introduced in 2014 in the United States and other countries. A survey was developed in 2016 to gauge dog owner adherence with veterinary recommendations around the administration of preventive flea and tick medications. Current fluralanerusing dog owners were also asked to compare their experience with opinions on monthly flea and tick products.

Aim: To survey dog owners who were current users of fluralaner on their opinions, experiences, and attitudes around the administration of flea and tick medications to their dogs in light of current veterinarian recommendations.

Methods: Dog owners in the United States (US), United Kingdom (UK), and Australia that gave fluralaner oral chews to their dogs were asked to compare their experience using fluralaner (12-week dosing) and monthly flea and tick medications. The survey responses of dog owners in the UK and Australia were compared against responses to a similar survey conducted in the US in 2017. Surveys were completed by dog owners who were in the clinic for any reason other than a sickness visit. Additionally, veterinarians that prescribed fluralaner from all three countries provided their annual flea and tick treatment recommendation for dogs.

Results: A sample of veterinarians from the US, UK, and Australia that prescribe fluralaner recommend that dog owners obtain approximately 12 months of flea protection per year and 9-12 months of tick protection per year. A variable proportion of owners (22\%-90\%) reported that their dog participates in outdoor and social activities associated with an increased flea and tick exposure risk. A similarly variable proportion of owners reported prior experience of finding fleas $(24 \%-50 \%)$ or ticks $(18 \%-35 \%)$ on their dogs. All participating owners treated their dogs currently with fluralaner and most $(68 \%-77 \%)$ had previously treated their dog with monthly flea and tick products. The convenience of 12-week dosing and less frequent dosing were the most frequently identified product qualities associated with their choice of an extended effect flea and tick treatment.

Conclusion: Most veterinarians surveyed in this survey recommended year-round use of a flea and tick medication for dogs in the US, UK, and Australia. Dog owners recalled the veterinary recommendation for flea and tick prevention as 8-10 months per year. Most dog owners from the clinics in the US, UK, and Australia had used shorter-acting (monthly) flea/tick medications previously. The majority of those who currently gave fluralaner doses to their dogs were "satisfied" or "very satisfied" with the extended duration flea and tick product. Preference for a 12-week duration medication over monthly re-treatment was also high $(82 \%-92 \%)$ in all three countries and was associated with convenience.
\end{abstract}

Keywords: Dogs, Ectoparasites, Fluralaner, Preference, Satisfaction.

\section{Introduction}

Fleas and ticks are common ectoparasites of animals that can cause disease by direct skin irritation and by transmitting pathogens or toxins while blood feeding (Shaw et al., 2001; Chomel, 2011; Yancey et al., 2014; Fisara and Webster, 2015; Lappin, 2018). Treatments are available to remove these parasites from cats and dogs and eliminate the juvenile life stages from homes and properties. In recent years, isoxazoline class ectoparasiticides have become available as an effective systemic treatment for fleas and ticks on cats and dogs (Pfister and Armstrong, 2016). Most ectoparasiticides for dogs and cats, including the isoxazolines, are dosed at a monthly retreatment interval; however, the novel oral isoxazoline fluralaner $\left(\right.$ Bravecto $^{\circledR}$, Merck \& Co., Inc., Kenilworth, NJ). is uniquely registered for administration to dogs with a 12-week retreatment interval in the US (Food and Drug Administration (FDA), 2014), UK, and Australia against fleas. The label of the oral Bravecto Chews product has a duration 
claim for ticks that differs from country to country. In the US, the label has a 12-week claim for Ixodes scapularis (black-legged tick), Dermacentor variabilis (American dog tick) and Rhipicephalus sanguineus (brown dog tick) and an 8-week claim for Amblyomma americanum (lone star tick). In the UK, the label has a 12-week claim for Ixodes Ricinus (castor bean tick), Dermacentor reticulatus (ornate dog tick) and Dermacentor variabilis (American dog tick) and an 8-week claim for Rhipicephalus sanguineus (brown dog tick). In Australia, the label has a 16-week claim for Ixodes holocyclus (paralysis tick) and an 8-week claim for Rhipicephalus sanguineous (brown dog tick) and Haemaphysalis longicornis (bush tick).

A prior survey demonstrated that pet owner adherence to veterinary recommendations for canine flea and tick protection in the US is limited (Lavan et al., 2017a). Another study showed on average, dog owners purchased approximately 5-6 months of flea and tick protection when using up to a 12 -week retreatment interval treatment but purchased 1-2 months less per year when using a flea and tick treatment with a monthly re-dosing interval (Lavan et al., 2018c). Furthermore, the change up to a 12 -week retreatment interval had no impact on dog owner adherence to heartworm prophylaxis because US owners purchased an average of just over 7 months of heartworm prophylaxis (Lavan et al., 2018b), regardless of the retreatment interval for concurrent flea and tick control. Among fluralaner-using dog owners, overall satisfaction with and preference for a 12 -week flea and tick medication compared to monthly medications was high in the U.S. (Lavan et al., 2017b) but unknown in other countries.

An additional two studies were conducted to evaluate the responses of dog owners in the UK and Australia regarding their flea and tick treatment preferences and to compare the results across the three countries. This paper presents the results of this 3-country comparison in fluralaner-using dog owners. Within the fluralaner-dispensing veterinary practices of which survey respondents were fluralaner-using clients, the proportion of dog owners who had used another flea and tick product was $68 \%-77 \%$ (Table 4, Question 1), but the proportion of owners who had used fluralaner and switched to another product or discontinued all flea and tick medication was not known.

The objectives of this survey were:

1. To sample veterinarian recommendations for flea and tick medication use in the UK and Australia and compare this with veterinary recommendations previously reported from the US (Lavan et al., 2017b). 2. To compare the survey responses of dog owners in the US (Lavan et al., 2017b) with dog owners from the UK and Australia who also used fluralaner for canine flea and tick prevention. Survey questions assessed satisfaction, convenience, dosing, and preference for or against fluralaner compared to monthly flea and tick medications as well as the product qualities they prioritize.

The monthly ectoparasiticides category includes older products (fipronil, pyrethroids, and imidacloprid) and the newer isoxazoline products. Dog owners were not asked which of these monthly products they had used in the past although their experience with these monthly products would influence their attitudes toward their fluralaner experience.

In our surveys of veterinarians in the US, UK, and Australia, we found that the vast majority of veterinarians surveyed recommended 12 months per year protection against fleas. Similarly, all three countries have a 12week label claim for flea efficacy which allows them to be compared relative to dog owner attitudes toward the oral fluralaner product.

\section{Materials and Methods}

The methodology for the 2017 survey in the US (Lavan et al., 2017b) was the basis for similar protocols followed in the UK and Australia. The MSD Animal Health sales force identified clinics in each region that prescribed fluralaner. The goal was to get $2-5$ clinics per countryspecific region for a minimum of 25 clinics per country. The study manager contacted a clinic point person at each clinic to assess their interest in participation. Following clinic enrollment, a short survey was sent to the managing veterinarian to obtain their annual flea and tick control recommendations. Each clinic had a goal to survey 20 current fluralaner-using dog owners. There were small differences between countries in questionnaire wording, as described below.

Clinics were not allowed to hand pick participants but were asked to choose one owner each day who was in the clinic for some other reason (wellness visit, pick up dog food, grooming, etc.). To be able to knowledgeably comment on their fluralaner experience, owners needed to have a dog that was currently receiving fluralaner, had purchased at least one or more doses for the dog in the past and not currently be participating in a health plan. Dog owners in health plans obtain their wellness visits, preventive services as well as a full year of heartworm, and flea/tick medication for the price of the plan, payable in 12 monthly installments. There was concern that they might be more favorably inclined toward the medications they receive through the plan. If a dog owner could not be identified that fit the inclusion criteria, the clinic staff could look at the appointment record for the previous day to identify someone who qualified and then call them at home. If no one was identified from the previous days' appointments, then they would move back in time one additional day and repeat the search.

At least 25 veterinary clinics in each country agreed to participate with at least one veterinarian in each clinic providing information about their ectoparasite prophylaxis recommendation. These recommendations were recorded separately for fleas and ticks. Paper 
questionnaires were completed in the waiting room or over the phone with the assistance of a clinic staff member. Staff members ensured that all survey questions were answered and that the dog owner understood each question.

The first page of the survey asked for demographic details for the owner and dog along with prior experiences observing fleas and ticks. The second page of the survey focused on questions related to dog owner experience with fluralaner and monthly flea/tick products. Survey questions focused on a single concept [satisfaction (Q2), convenience (Q9), preference (Q10)] or tested a group of concepts that had previously been identified in small focus groups of dog owners (Q3, Q4, and Q5). Questions 6, 7, and 8 examined aspects of the extended duration dosing of fluralaner to assess whether dog owners struggled to comply with 12 -week re-dosing.

Owners also completed a series of questions to gain an in-depth assessment of their opinions on flea and tick treatment. The questions were designed to particularly focus on the owners' experiences with the extended 12-week retreatment interval of fluralaner compared with the monthly retreatment interval of other commonly used flea and tick treatments. Similar survey methodologies were used in the three countries; however, the exact survey questionnaire wording was appropriately modified as necessary to be understandable to pet owners. These differences in wording among the different country surveys were minimal and were made to adapt to local phrasing or customary pet owner behavior for each country. For example, US owners recognize "dog park" as an outdoor place where dogs frequently mingle, while this term was not as commonly used in the UK at the time. Therefore, a US question referencing "dog parks" was substituted to reference "boarding kennel" for UK and AUS owners. Surveys underwent cognitive testing with dog owners as they were developed in order to improve comprehension.

Another difference among the surveys relates to different parasite risks when assessing pet owner recall of the veterinary recommendation on flea and tick protection. In the US and UK, owners were asked for a single amount of time (months per year) to protect against both fleas and ticks. However, in Australia, the Paralysis Tick (I. holocyclus) represents a very serious health hazard in specific geographic areas, and owners were asked for a separate response on the recommended duration of protection against fleas or Paralysis Ticks. The data were pooled from all of the regions in the respective countries to gather a national average. The analysis by the region within a country was not done, due to the limited numbers of dogs and owners per region.

\section{Ethical approval}

Ethical approval was not sought for this study because no live animals were used. All responding pet owners provided signed consent to complete the survey.

\section{Results}

Veterinarians (26 US, 35 UK, 28 AUS) from participating clinics provided their annual flea and tick control recommendation for dogs (Table 1). The number of participating veterinarians, their years as practicing clinicians and the distribution of the flea/tick medication recommendations are included in Table 1.

Owners provided information on 1,608 dogs as summarized in Table 2. The number of owners who completed surveys varied between countries because of the relative success that clinics had in finding eligible dog owners who agreed to participate. On average, there were slightly more male dogs $(51 \%)$ than female dogs $(49 \%)$ and most dogs $(86 \%)$ were neutered. The average dog was approximately $18 \mathrm{~kg}$ in body weight and approximately 5 years of age. Many dogs in the three countries participated in outdoor activities, averaging 3-4 hours in the US and UK but averaging over 8 hours in Australia which helps characterize the potential risk for flea or tick exposure. Most dog owners characterized their dogs as being in good or excellent health. Canine participation in selected outdoor activities is listed in Table 2. These also help characterize the dog's flea and tick exposure.

Table 1. Clinical practice experience of veterinarians and their flea/tick control recommendations in three different countries.

\begin{tabular}{|c|c|c|c|c|}
\hline & & United States $^{\mathrm{a}}$ & United Kingdom & Australia \\
\hline \multicolumn{2}{|c|}{ Participating veterinarians } & 24 & 35 & 28 \\
\hline \multicolumn{2}{|c|}{ Years in practice $($ Mean \pm SD) } & $17.4 \pm 10.7$ & $12.0 \pm 10.6$ & $19.5 \pm 11.5$ \\
\hline \multicolumn{5}{|c|}{$\begin{array}{l}\text { Number of veterinarians with a particular } \\
\text { flea or tick recommendation }[\%(n)]\end{array}$} \\
\hline \multirow{2}{*}{ Fleas } & 12 months & $96 \%(23)$ & $97 \%(34)$ & $100 \%(28)$ \\
\hline & Less than 12 months & $3 \%(1)$ & $3 \%(1)$ & 0 \\
\hline \multirow{2}{*}{ Ticks } & 12 months & $96 \%(23)$ & $49 \%(17)$ & $75 \%(21)$ \\
\hline & Less than 12 months & $3 \%(1)$ & $51 \%(18)$ & $25 \%(7)$ \\
\hline
\end{tabular}

${ }^{a}$ Lavan et al. (2017b). 
Table 2. Owner descriptions of their dogs in US, UK, and Australia ${ }^{a}$.

\begin{tabular}{|c|c|c|c|}
\hline & United States $^{b}$ & United Kingdom & Australia \\
\hline Number of owners completing questionnaire & 559 & 537 & 512 \\
\hline \multicolumn{4}{|l|}{ Dog gender $[\%(n)]$} \\
\hline Male & $51.6 \%(288)$ & $50.1 \%(269)$ & $52.3 \%(268)$ \\
\hline Female & $48.4 \%(270)$ & $49.5 \%(266)$ & $47.7 \%(244)$ \\
\hline \multicolumn{4}{|l|}{ Dog neuter status $[\%(n)]$} \\
\hline Neutered & $88.8 \%(496)$ & $76.9 \%(413)$ & $90.8 \%(465)$ \\
\hline Intact & $11.2 \%(63)$ & $23.1 \%(124)$ & $9.2 \%(47)$ \\
\hline Dog body weight (Mean \pm SD) $\mathrm{kg}$ & $18.8 \pm 14.2$ & $19.3 \pm 11.9$ & $17.2 \pm 11.9$ \\
\hline Dog age $($ Mean $\pm \mathrm{SD})$ years & $6.1 \pm 3.6$ & $5.1 \pm 3.6$ & $5.3 \pm 4.0$ \\
\hline Mean hours outdoors per day (Mean \pm SD) & $4.2 \pm 4.9$ & $3.7 \pm 2.7$ & $8.3 \pm 6.6$ \\
\hline \multicolumn{4}{|l|}{ How healthy is your dog? $[\%(n)]$} \\
\hline Excellent & $58.9 \%(329)$ & $54.7 \%(294)$ & $58.7 \%(301)$ \\
\hline Good & $34.8 \%(194)$ & $39.3 \%(211)$ & $35.0 \%(179)$ \\
\hline Fair & $6.0 \%(34)$ & $5.7 \%(31)$ & $5.9 \%(30)$ \\
\hline Poor & $0.4 \%(2)$ & $0.2 \%(1)$ & $0.4 \%(2)$ \\
\hline \multicolumn{4}{|c|}{ List your dog's activities (Check all that apply) $[\%(n)]$} \\
\hline Dog park & $32.5 \%(182)$ & $\mathrm{n} / \mathrm{a}$ & $\mathrm{n} / \mathrm{a}$ \\
\hline Swims & $24.9 \%(139)$ & $43.4 \%(233)$ & $45.1 \%(231)$ \\
\hline Walks off leash & $48.8 \%(273)$ & $82.5 \%(443)$ & $65.2 \%(334)$ \\
\hline Access to woods/bush/national parks & $39.7 \%(222)$ & $81.4 \%(437)$ & $40.8 \%(209)$ \\
\hline Access to high or uncut grass & $51.7 \%(289)$ & $80.1 \%(430)$ & $50.0 \%(256)$ \\
\hline Socializes with other dogs & $\mathrm{n} / \mathrm{a}$ & $90.5 \%(486)$ & $85.7 \%(439)$ \\
\hline Boarded in kennels & $\mathrm{n} / \mathrm{a}$ & $22.0 \%(118)$ & $31.3 \%(160)$ \\
\hline
\end{tabular}

${ }^{a}$ Totals include all known answers and do not include missing data.

bLavan et al. (2017b).

Over 500 dog owners completed surveys in each of the three countries (Table 3). Approximately, $74 \%$ of participants were female and most $(64 \%)$ were in the 30-59-year age block. Dog owners had been caretakers for their animals for an average of 4-5 years. Many dog owners who participated in these three studies had prior direct experience with fleas and ticks and most $(69 \%-$ $77 \%$ ) had used other flea/tick medications before using fluralaner (Table 4, Question 1).

In this survey, veterinarians recommended nearly 12 months of protection against fleas and 9-12 months for ticks. Approximately, one third of owners could not recall their veterinarian's recommendation on flea and tick control. The owners who could recall this recommendation underestimated the duration, based on 12 months of flea protection, by more than 1 month (1.2 months less in the US, 3.4 months less in the UK, and 1.3 months less in AUS). The owners' opinion was that protection is needed for most of the year although typically shorter than the duration recommended by veterinarians.
The dog owner survey contained 10 multiple choice questions which asked about the dog owner's experience with fluralaner as well as non-fluralaner flea/ tick medications. Many questions offered an additional response choice that allowed the owner to write in their own free text response. The wording of each question and the distribution of answers by country is shown in Table 4.

Question 1, which asked about prior experience with monthly flea and tick products, allowed the creation of a subgroup that could compare their experience with both fluralaner and monthly products. Question 2 asked about the fluralaner-using dog owner's level of satisfaction with the canine fluralaner oral chews. Questions 3, 4, and 5 were developed in 2016 from the responses of fluralaner-using dog owners in small focus groups in the US. The use of a three-country survey was an opportunity to assess whether or not the qualities identified in the small groups resonated with a larger group of fluralaner-using dog owners. Questions 6 , 7, and 8 all tried to assess different aspects of $12-$ week dosing and whether it was difficult to adhere to 
Table 3. Dog owner demographics and reported ectoparasite observation experience in US, UK, and Australia ${ }^{\mathrm{a}}$.

\begin{tabular}{|c|c|c|c|c|}
\hline & $\begin{array}{l}\text { United } \\
\text { States }^{\mathrm{b}}\end{array}$ & $\begin{array}{c}\text { United } \\
\text { Kingdom }\end{array}$ & \multicolumn{2}{|c|}{ Australia } \\
\hline Number of owners completing questionnaire & 559 & 537 & \multicolumn{2}{|c|}{512} \\
\hline \multicolumn{5}{|l|}{ Owner gender $[\%(n)]$} \\
\hline Male & $26.4 \%(140)$ & $24.9 \%(129)$ & \multicolumn{2}{|c|}{$25.8 \%(129)$} \\
\hline Female & $73.6 \%(390)$ & $75.1 \%(389)$ & \multicolumn{2}{|c|}{$74.2 \%(371)$} \\
\hline \multicolumn{5}{|l|}{ Owner Age Range [\% $(n)]$} \\
\hline $10-29$ & $9.7 \%(48)$ & $10.7 \%(52)$ & \multicolumn{2}{|c|}{$19.0 \%(93)$} \\
\hline $30-59$ & $63.7 \%(316)$ & $65.9 \%(321)$ & \multicolumn{2}{|c|}{$64.6 \%(316)$} \\
\hline $60-89$ & $26.6 \%(132)$ & $23.4 \%(114)$ & \multicolumn{2}{|c|}{$16.4 \%(80)$} \\
\hline $\begin{array}{l}\text { Mean years as primary caregiver for this dog } \\
(\text { Mean } \pm \text { SD) }\end{array}$ & $5.5 \pm 3.6$ & $4.5 \pm 3.4$ & \multicolumn{2}{|c|}{$4.7 \pm 3.5$} \\
\hline \multicolumn{5}{|l|}{ Have you ever seen fleas? [\% $(n)]$} \\
\hline On this dog & $41.1 \%(230)$ & $24.4 \%(131)$ & \multicolumn{2}{|c|}{$48.6 \%(249)$} \\
\hline In your house & $11.8 \%(66)$ & $11.7 \%(63)$ & \multicolumn{2}{|c|}{$18.8 \%(96)$} \\
\hline In your bed(s) & $3.2 \%(18)$ & $2.4 \%(13)$ & \multicolumn{2}{|c|}{$2.7 \%(14)$} \\
\hline On your family & $2.7 \%(15)$ & $3.7 \%(20)$ & \multicolumn{2}{|c|}{$2.7 \%(14)$} \\
\hline On other pets & $\mathrm{n} / \mathrm{a}$ & $\mathrm{n} / \mathrm{a}$ & \multicolumn{2}{|c|}{$16.2 \%(83)$} \\
\hline \multicolumn{5}{|l|}{ Have you ever seen ticks? [\% $(n)]$} \\
\hline On this dog & $31.3 \%(175)$ & $34.8 \%(187)$ & \multicolumn{2}{|c|}{$18.0 \%(92)$} \\
\hline In your house & $5.7 \%(32)$ & $5.4 \%(29)$ & \multicolumn{2}{|c|}{$2.0 \%(10)$} \\
\hline In your bed(s) & $2.3 \%(13)$ & $0.6 \%(3)$ & \multicolumn{2}{|r|}{0} \\
\hline On your family & $10.7 \%(60)$ & $6.3 \%(34)$ & \multicolumn{2}{|c|}{$9.0 \%(46)$} \\
\hline On other pets & $\mathrm{n} / \mathrm{a}$ & $\mathrm{n} / \mathrm{a}$ & \multicolumn{2}{|c|}{$7.2 \%(37)$} \\
\hline $\begin{array}{l}\text { Mean recalled duration of veterinarian's flea and tick } \\
\text { protection recommendation (months/year) }\end{array}$ & $10.8 \pm 2.8$ & $8.7 \pm 4.2$ & $\begin{array}{l}10.7 \pm 3.0 \\
\text { (fleas) }\end{array}$ & $\begin{array}{c}9.6 \pm 3.4 \\
\text { (paralysis tick) }\end{array}$ \\
\hline Number of pet owners with no idea $[\%(n)]$ & $25.4 \%(137)$ & $22.0 \%(118)$ & $28.7 \%(147)$ & $35.4 \%(181)$ \\
\hline $\begin{array}{l}\text { Mean owner opinion on flea and tick protection needed } \\
\text { (months/year) }\end{array}$ & $10.5 \pm 2.7$ & $9.3 \pm 3.4$ & $\begin{array}{l}10.6 \pm 2.9 \\
\text { (fleas) }\end{array}$ & $\begin{array}{c}9.6 \pm 3.6 \\
\text { (paralysis tick) }\end{array}$ \\
\hline
\end{tabular}

${ }^{a}$ Totals include all known answers and do not include missing data. ${ }^{\text {bLavan }}$ et al. (2017b).

12-week dosing. Question 9 and 10 asked about the dog owner's perception of the convenience or preference for or against fluralaner, compared to monthly flea/tick products.

\section{Discussion}

The surveys from the UK and Australia demonstrated that veterinarian opinions regarding flea and tick control are very similar in clinics in all three countries that prescribe fluralaner. In this survey, most veterinarians recommended 12 months of flea control per year. The oral (chew) form of fluralaner is available in all three countries and labeled to protect against fleas for 12 weeks.

In the United States, relative to Australia and the UK, the higher proportion of veterinarians recommending
12 months of tick control may reflect the perceived threat of tick-borne disease at the time of the survey. The Paralysis Tick risk in Australia is seasonal and not evenly distributed across the country, and this may alter the perception of tick protection that is required in different regions of that country. UK veterinarians were the least likely to recommend 12 months of tick protection (49\% of UK veterinary responders) although UK dog owners were the most likely to report ticks on the dog (34\%) versus US (31\%) and Australian $(18 \%)$ dog owners. Fluralaner is available in the US and UK by prescription only but is an over-the-counter medication in Australia.

The survey objectives guided the survey design, which created a source of bias. In order to gather dog owner opinions on their experience providing fluralaner to their 
Table 4. Dog owner flea- and tick-control survey questions and responses in the US, UK, and Australia Studies.

\begin{tabular}{|c|c|c|c|}
\hline & United States $^{\mathrm{a}}$ & United Kingdom & Australia \\
\hline Dates of survey & Mar-May, 2016 & Aug-Oct, 2016 & Jan-Mar, 2017 \\
\hline \multicolumn{4}{|c|}{ 1. Other than Bravecto, have you used any products to kill fleas/ticks or protect your dog from fleas/ticks in the past? } \\
\hline Yes & $72.6 \%$ & $68.0 \%$ & $76.6 \%$ \\
\hline No & $19.5 \%$ & $29.2 \%$ & $18.9 \%$ \\
\hline \multicolumn{4}{|c|}{ 2. Please circle your level of satisfaction with Bravecto. } \\
\hline Very Satisfied & $66.4 \%$ & $70.6 \%$ & $60.5 \%$ \\
\hline Satisfied & $29.6 \%$ & $25.9 \%$ & $33.8 \%$ \\
\hline Neither satisfied nor unsatisfied & $3.4 \%$ & $2.0 \%$ & $3.1 \%$ \\
\hline Unsatisfied & $0.6 \%$ & $0.6 \%$ & $0.8 \%$ \\
\hline Very Unsatisfied & $0.0 \%$ & $0.4 \%$ & $0.8 \%$ \\
\hline \multicolumn{4}{|c|}{ 3. Which of the following Bravecto benefits are important to you as a pet owner? Circle all that apply. } \\
\hline Convenience & $72.5 \%$ & $73.0 \%$ & $69.1 \%$ \\
\hline 12-week dosing & $66.7 \%$ & $67.2 \%$ & $78.1 \%$ \\
\hline Dosing less often & $66.5 \%$ & $48.2 \%$ & $51.0 \%$ \\
\hline How quickly it kills fleas and ticks & $45.3 \%$ & $50.8 \%$ & $43.4 \%$ \\
\hline Easier dosing puts less stress on me & $44.9 \%$ & $39.9 \%$ & $40.2 \%$ \\
\hline Palatability & $42.9 \%$ & $51.2 \%$ & $52.9 \%$ \\
\hline Other (free text) & $6.1 \%$ & $6.5 \%$ & $6.3 \%$ \\
\hline \multicolumn{4}{|c|}{ 4. If you had to select ONE, which one is the most important reason to use Bravecto? Please circle one. } \\
\hline 12-week dosing & $25.0 \%$ & $25.9 \%$ & $41.0 \%$ \\
\hline Convenience & $20.6 \%$ & $21.6 \%$ & $16.7 \%$ \\
\hline Dosing less often & $19.2 \%$ & $11.1 \%$ & $10.7 \%$ \\
\hline How quickly it kills fleas and ticks & $18.4 \%$ & $23.7 \%$ & $19.7 \%$ \\
\hline Easier dosing puts less stress on me & $8.6 \%$ & $5.1 \%$ & $4.3 \%$ \\
\hline Palatability & $4.8 \%$ & $8.1 \%$ & $3.2 \%$ \\
\hline Other (free text) & $3.2 \%$ & $4.5 \%$ & $4.5 \%$ \\
\hline
\end{tabular}

5. In your opinion, does using a product that lasts up to 12 weeks have an advantage over flea/tick products that require monthly re-dosing? Circle all that apply.

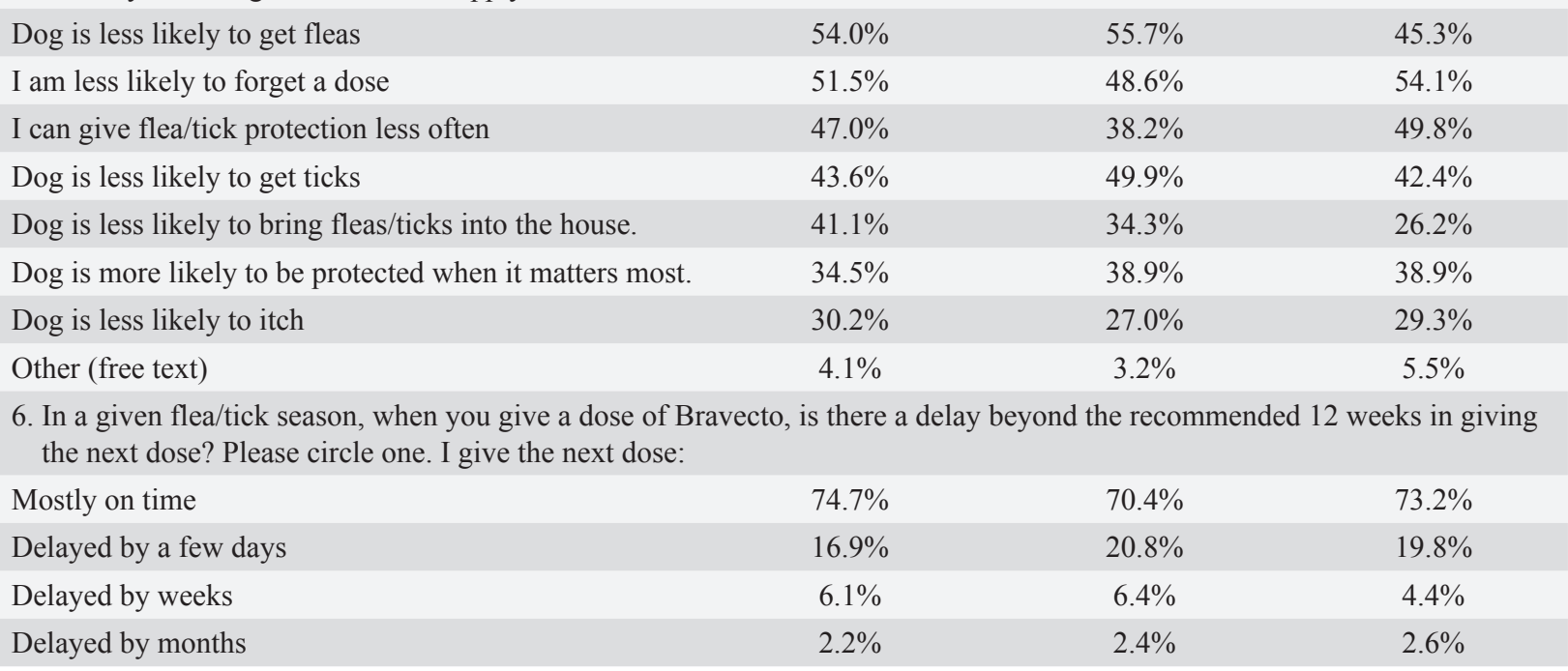




\begin{tabular}{|c|c|c|c|}
\hline & United States $^{\mathrm{a}}$ & United Kingdom & Australia \\
\hline \multicolumn{4}{|c|}{$\begin{array}{l}\text { 7. Are you more likely to give the next Bravecto dose on time compared to giving the repeat doses of monthly flea and tick } \\
\text { products? Please circle one, }\end{array}$} \\
\hline Yes & $64.6 \%$ & $73.6 \%$ & $63.2 \%$ \\
\hline No & $4.0 \%$ & $3.6 \%$ & $2.8 \%$ \\
\hline About the same & $26.8 \%$ & $20.8 \%$ & $25.7 \%$ \\
\hline I don’t know & $4.7 \%$ & $2.1 \%$ & $8.3 \%$ \\
\hline \multicolumn{4}{|c|}{ 8. With Bravecto, dog has (fewer months/same number of months/more months) of flea/tick protection in a year. Please circle on } \\
\hline Fewer months of protection & $7.8 \%$ & $4.3 \%$ & $5.2 \%$ \\
\hline Same number of months of protection & $56.4 \%$ & $50.5 \%$ & $56.0 \%$ \\
\hline More months of protection & $35.8 \%$ & $45.3 \%$ & $38.9 \%$ \\
\hline \multicolumn{4}{|c|}{ 9. Is it more convenient to give repeat doses of Bravecto than to give repeat doses of a monthly flea/tick product? } \\
\hline More convenient & $88.7 \%$ & $87.8 \%$ & $87.5 \%$ \\
\hline Same convenience & $10.2 \%$ & $12.2 \%$ & $11.5 \%$ \\
\hline Less convenient & $1.0 \%$ & 0 & $1.0 \%$ \\
\hline \multicolumn{4}{|c|}{ 10. Do you prefer Bravecto over other flea and tick products that you have used? Please circle one. } \\
\hline Yes & $89.0 \%$ & $91.7 \%$ & $82.4 \%$ \\
\hline No & $1.3 \%$ & $1.2 \%$ & $3.8 \%$ \\
\hline About the same & $9.7 \%$ & $7.1 \%$ & $13.8 \%$ \\
\hline
\end{tabular}

${ }^{a}$ Lavan et al. (2017b).

dogs, dog owners were surveyed in clinics that were currently prescribing the extended duration fluralaner chew at the time that the survey was conducted. All of the survey respondents were currently giving fluralaner chews to their dogs. Clinics were selected because they were currently prescribing fluralaner to canine patients. Pet owners may have used other flea and tick products, but the questions directly targeted their experience with fluralaner.

Pet owners would not be expected to remember the veterinary recommendation for flea and tick prevention if it is not frequently emphasized during communication opportunities between the pet owner and the clinic staff. In this survey, dog owners recalled the veterinary recommendation for flea and tick protection as 1-2 fewer months than the actual recommendation reported by veterinarians in each country (Table 3 ). Approximately, $25 \%$ of dog owners had no recollection of what the veterinary recommendation might be although all these owners had experience administering flea/tick products. When dog owners were asked for their opinion regarding the number of months of flea/ tick protection their dog needed each year (Table 3), the estimates were similar to the number of months that the pet owner erroneously recalled for the veterinary recommendation (9-10 months). This survey did not attempt to assess the dog owner's behavior (purchases of flea and tick medication in response to the veterinary recommendation) although other studies have looked at this in the US (Lavan et al., 2018c) and Spain (Lavan et al., 2018a). These studies both found that the average dog owner who purchased a flea/tick medication for their dog actually went home with 6 months or less of protection in a 12 -month period.

The need for flea/tick medication for dogs was illustrated by the number of dogs that participated in outside activities (Table 2) and dog owners who had direct experience with fleas and/or ticks (Table 3). Australian dog owners reported that their average dog was outside nearly twice as long as the US and UK dogs ( 8 hours vs. 4 hours). Australian dog owners were also more likely to report fleas on the $\operatorname{dog}(48 \%)$ versus dogs in the US (41\%) and UK (24\%). They were also nearly twice as likely to report fleas in the house (18\%) as the UK or US (both $11 \%$ ). Most dog owners who were currently giving fluralaner doses to their dogs (over 93\%) in all three countries expressed satisfaction with their fluralaner experience (Table 4, question 2). Less than $5 \%$ of owners in any of the three countries expressed dissatisfaction with the extended duration flea/tick medication, although the reasons for this dissatisfaction were not explored.

When asked to choose one product feature that was most important to them (question 4, Table 4), dog owners who were currently giving fluralaner doses to their dogs chose "12-week dosing" most frequently. This feature clearly sets fluralaner apart from other flea and tick products. The product feature which was the second choice of owners in the UK and AUS ["how quickly (fluralaner) kills fleas and ticks] focused on efficacy which may be related to the perceived risk of flea and tick exposure from outdoor locations (UK) or 
the amount of time that the dog is outside on an average day (AUS).

The benefits of using an extended duration flea and tick medication (question 5, Table 4) again focused on measures of convenience and efficacy in the three countries. Fluralaner-using owners in the US and UK most often selected "dog is less likely to get fleas" as the biggest benefit, indicating a focus on flea infestation prevention. UK owners took that further by selecting "dog is less likely to get ticks" as the second ranked benefit which may reflect the frequency with which they reported seeing ticks on the dog (Table 3). Dog owners in Australia selected their top benefits around convenience ("less likely to forget a dose" and "give flea/tick protection less often"). These responses, along with the dog owner responses to the questions on satisfaction (Q2) and preference (Q10), may also reflect the property of a longer-acting flea/tick medication which requires fewer doses per year and provides more months of protection in each dose compared to monthly treatments.

Questions 6, 7, and 8 all asked about the difficulty of delivering fluralaner doses on a 12-week dosing schedule. Most fluralaner-using dog owners $(70 \%-$ $74 \%$ ) in the US, UK, and AUS studies felt that they could re-dose fluralaner "on time" or would be delayed by a few days $(16 \%-20 \%, \mathrm{Q} 6)$. When fluralaner-using owners were asked whether they were more likely to give doses of the 12-week fluralaner on time compared to giving doses of monthly products (question 7), $63 \%-73 \%$ of owners in all three countries responded favorably, again suggesting that the dog owners who used fluralaner did not struggle to dose on time. The results suggest that dog owners who use fluralaner do not feel that a 12-week dosing interval makes them more likely to forget dosing versus a monthly regimen. The dog owners who thought that the re-dose might be delayed by weeks or months amounted to $6 \%-8 \%$ of responders $(\mathrm{Q} 6)$. It is important to keep in mind that this question has a potential for bias. Owners do not want to look like bad caretakers and might overestimate their dosing compliance by boosting their estimate of "on-time" dosing.

The benefits of a longer medication duration were associated in previous studies in Spain and the US with the owner purchasing more months of coverage per year for dogs receiving fluralaner compared to monthly flea/ tick products (Lavan et al., 2018a, 2018c). In Spain, dog owners acquired $48 \%$ more months of coverage per year with fluralaner than with one of 19 monthly topical products, or $34 \%$ more months per year compared with 3 monthly oral products (Lavan et al., 2018a). In the US, dog owners who purchased fluralaner acquired $24 \%-73 \%$ more months of coverage per year when compared to two specific oral monthly flea/tick medications (Lavan et al., 2018c). In both studies, dog owners who chose fluralaner were significantly less likely to purchase 1-6 months and significantly more likely to purchase 7-12 months of flea and tick medication per year, leading to increased compliance with veterinary recommendations.

\section{Acknowledgments}

The authors wish to acknowledge Amy Pavlock (AMP Research Solutions) and Jane Fletcher (Think Smart, LLC) for their help in conducting the studies in the US and UK. We would also like to acknowledge Dongmu Zhang, Qian Xia, and Julia Feng for providing analysis and statistical support.

Data were obtained under a data-sharing contract. No live animals were used in this study and medical records were not examined. All transaction records were blinded as to the clinic identity, pet owner's identity and home address.

\section{Conflict of interest}

All authors are employees of MSD Animal Health. Study funding was provided by MSD Animal Health.

\section{Author's contribution}

All of the authors participated in conducting the studies in one or more countries and contributed to drafting the manuscript.

\section{References}

Chomel, B. 2011. Tick-borne infections in dogs - an emerging infectious threat. Vet. Parasitol. 179, 294-301.

Fisara, P. and Webster, M. 2015. A randomized controlled trial of the efficacy of orally administered fluralaner (Bravecto ${ }^{\mathrm{TM}}$ ) against induced Ixodes holocyclus (Australian paralysis tick) infestations on dogs. Parasit. Vector. 8, 257.

Food and Drug Administration (FDA). 2014. Freedom of Information Summary. Original New Animal Drug Application (NADA 141-426). Kenilworth, NJ: BRAVECTO Chews, .

Lappin, M. 2018. Update on flea and tick associated diseases of cats. Vet. Parasitol. 254, 26-9.

Lavan R., Armstrong, R., Burgio, F. and Tunceli, K. 2018a. Duration of annual flea and tick protection provided by dog owners in Spain. Parasit. Vector. $11,458-65$.

Lavan R., Armstrong, R., Normile, D., Zhang, D. and Tunceli, K. 2017b. Results from a U.S. dog owner survey on the treatment satisfaction and preference for fluralaner against flea and tick infestations. J. Vet. Sci. Technol. 8, 3; doi:10.4262/21577579.1000439.

Lavan, R.P., Armstrong, R., Tunceli, K. and Normile, D. 2018c. Dog owner flea/tick medication purchases in the USA. Parasit. Vector. 11, 581.

Lavan, R.P., Heaney, K., Vaduvoor, S.R. and Tunceli, K. 2018b. A comparative analysis of heartworm medication use patterns for dogs that also receive ectoparasiticides. Parasit. Vector. 11, 493-7.

Lavan, R.P., Tunceli, K., Zhang, D., Normile, D. and Armstrong, R. 2017a. Assessment of dog owner 
adherence to veterinarians' flea and tick prevention recommendations in the United States using a cross-sectional survey. Parasit. Vector. 10, 284-90.

Pfister, K. and Armstrong, R. 2016. Systemically and cutaneously distributed ectoparasiticides: a review of the efficacy against ticks and fleas on dogs. Parasit. Vector. 9, 436-50.

Shaw, S.E., Day, M.J., Birtles, R.J. and Breitschwerdt, E.B. 2001. Tick-borne infectious diseases of dogs. Trends Parasitol. 17, 74-80.
Yancey, C.B., Hegarty, B.C., Quriolo, B.A., Levy, M.G., Birkenheuer, A.J., Weber, D.J., Diniz, P. and Breitschwerdt, E.B. 2014. Regional seroreactivity and vector-borne disease co-exposures in dogs in the United States from 2004-2010: utility of canine surveillance. Vector Borne Zoonot. Dis. 14, 72432. 